\title{
THE EQUATIONS OF GRAND UNIFIED FIELD THEORY IN TERMS OF THE MAURER-CARTAN STRUCTURE RELATIONS OF DIFFERENTIAL GEOMETRY
}

\author{
Myron W. Evans \\ Alpha Institute for Advanced Study (AIAS) \\ E-mail: emyrone@aol.com
}

Received 9 June 2003

The first and second Maurer-Cartan structure relations are combined with the Evans field equation [1] for differential forms to build a grand unified field theory based on differential geometry. The tetrad or vielbein plays a central role in this theory, and all four fields currently thought to exist in nature can be described by the same equations, the tangent space index of the tetrad in general relativity being identified with the tetrad's internal (gauge group) index in gauge theory.

Key words: grand unified field theory, quantum grand unified field theory, Maurer-Cartan structure relations, differential geometry, $O(3)$ electrodynamics, $\mathbf{B}^{(3)}$ field.

\section{INTRODUCTION}

It is currently thought that there exist four fundamental force fields in nature: gravitational, electromagnetic, weak and strong. The development of a theory of all known force fields is one which goes back to the mid-nineteenth century, when attempts were made to unify the gravitational and electromagnetic fields. The contemporary theory of all four fields is known as "grand unified field theory" (GUFT). Recently a generally covariant, classical field/matter equation [1] and wave equation of quantum field/matter theory [2] was developed and applied to GUFT. In this theory the tangent bundle of generally relativity [3] is also the fiber bundle of gauge theory [4]. Both the tangent and fiber bundles are described [2] by the tetrad (or vielbein) $q_{\mu}^{a}[3]$ whose orthonormal tangent space index $a$ becomes the index both of the tangent space in general relativity and of the fiber bundle space in gauge theory. 
The index $\mu$ of the tetrad is that of the non-Euclidean base manifold. In general relativity, the base manifold is non-Euclidean space-time in four dimensions. GUFT is thereby developed [1,2] as a theory of general relativity [3], in which all the equations of physics are generally covariant. In quantum GUFT [2] the tetrad is the eigenfunction of the generally covariant wave equation [2] from which is obtained the equations of the quantized force and matter fields in nature. Examples of such equations in classical field/matter theory are the Einstein field equation and the equations of generally covariant electrodynamics [5-10], namely the $O(3)$ electrodynamic field/matter equations. These were obtained from the classical equation introduced in Ref. [1]. Equations of quantum field/matter theory such as the single particle wave equation, the Schrödinger equation, the Klein-Gordon and Dirac equations, were obtained from the quantized equation in Ref. [2]. The latter is a generally covariant, second-order wave equation which also gives wave and quantum field equations in higher symmetry electrodynamics [5-10], the $O(3)$ d'Alembert and Proca equations. Electrodynamics is thereby developed into a generally covariant theory, as required by the principle of general relativity [11]. The generally covariant wave equation [2] was used as a theory of the weak and strong fields by recognizing that the internal index of the tetrad is both the fiber bundle index of gauge field theory [3] and the tangent space index of general relativity. The internal gauge group index of the parity-violating weak field [4] therefore becomes a manifestation of geometry and is closely related to the internal $O(3)$ symmetry gauge group index $a=(1),(2),(3)$ of $O(3)$ electrodynamics, where $((1),(2),(3))$ denotes the orthonormal, $O(3)$ symmetry, complex circular basis of three-dimensional Euclidean space [5-10]. By developing the basis $((1),(2),(3))$ with an $S U(3)$ representation it becomes possible to apply the generally covariant classical [1] and quantum [2] equations into equations in the strong field [4].

In this theory, physics is reduced to geometry. The tetrad is used $[2,3]$ to introduce differential geometry into general relativity, and in this Letter the classical and quantum equations developed in Refs. [1] and [2] are combined with the Maurer-Cartan structure relations to give a GUFT theory based entirely on differential geometry [3]. In so doing, it is seen that all four fields are manifestations of differential geometry and can be inter-related with the structure relations combined with the Evans equations $[1,2]$ expressed as equations in differential forms $[3,4]$. If the index a represents the tangent space of the base manifold representing gravitation then the Evans equations [1,2] are those of gravitation. If the index $a$ has an $O(3)$ symmetry with the basis $((1),(2),(3))$, the Evans equations are those of generally covariant, or higher symmetry, electromagnetism $[1,2,5-10]$. If the index $a$ has an $S U(2)$ symmetry, the Evans equations are those of the parity-violating weak field; and if the index $a$ has $S U(3)$ symmetry, the Evans equations are those of the strong field. In order to build the theory from first geometrical principles Sec. 2 gives a description of the tetrad at 
work in $O(3)$ electrodynamics, and Sec. 3 gives the Evans equations as equations between differential forms and combines them with the Maurer-Cartan structure relations.

\section{THE TETRAD IN GENERALLY COVARIANT $(\mathrm{O}(3))$ ELECTRODYNAMICS}

Define the base manifold in general as the non-Euclidean spacetime indexed by $\mu=(0,1,2,3)$. In this manifold define the infinitesimal displacement vector in the space basis $\left(u_{1}, u_{2}, u_{3}\right)$ :

$$
d \mathbf{r}=\frac{\partial \mathbf{r}}{\partial u_{1}} d u_{1}+\frac{\partial \mathbf{r}}{\partial u_{2}} d u_{2}+\frac{\partial \mathbf{r}}{\partial u_{3}} d u_{3}
$$

and the three metric vectors $[2,9,11]$ :

$$
\mathbf{q}^{1}=\frac{\partial \mathbf{r}}{\partial u_{1}}, \quad \mathbf{q}^{2}=\frac{\partial \mathbf{r}}{\partial u_{2}}, \quad \mathbf{q}^{3}=\frac{\partial \mathbf{r}}{\partial u_{3}}
$$

Define the orthonormal tangent spacetime by the index $a$ of the $O(3)$ symmetry complex circular basis:

$$
\mathbf{e}^{(1)} \times \mathbf{e}^{(2)}=i \mathbf{e}^{(3) *} \text { et cyclicum, }
$$

whose unit vectors are related to the orthonormal Cartesian unit vectors $\mathbf{i}, \mathbf{j}, \mathbf{k}$ of the tangent space by

$$
\mathbf{e}^{(1)}=\frac{1}{\sqrt{2}}(\mathbf{i}-i \mathbf{j}), \quad \mathbf{e}^{(2)}=\frac{1}{\sqrt{2}}(\mathbf{i}+i \mathbf{j}), \quad \mathbf{e}^{(3)}=\mathbf{k} .
$$

In the tangent space, the displacement vector is

$$
d \mathbf{r}=\frac{\partial \mathbf{r}}{\partial e^{(1)}} d e^{(1)}+\frac{\partial \mathbf{r}}{\partial e^{(2)}} d e^{(2)}+\frac{\partial \mathbf{r}}{\partial e^{(3)}} d e^{(3)},
$$

and the three orthonormal metric vectors in the tangent space are

$$
\begin{gathered}
\mathbf{q}^{(1)}=\partial \mathbf{r} / \partial e^{(1)}=\mathbf{e}^{(1)}, \\
\mathbf{q}^{(2)}=\partial \mathbf{r} / \partial e^{(2)}=\mathbf{e}^{(2)}, \\
\mathbf{q}^{(3)}=\partial \mathbf{r} / \partial e^{(3)}=\mathbf{e}^{(3)} .
\end{gathered}
$$

Let the orthonormal tangent space rotate and translate with respect to the space $i=1,2,3$ by introducing the phase $\phi=\omega t-\kappa Z$ of the 
wave equation [5-10]. Here $\omega$ is an angular frequency at instant $t$ and $\kappa$ is a wave-vector at point $\mathrm{Z}$. The orthonormal tangent space is thereby defined by the metric vectors

$$
\mathbf{q}^{(1)}=\mathbf{e}^{(1)} e^{i \phi}, \quad \mathbf{q}^{(2)}=\mathbf{e}^{(2)} e^{-i \phi}, \quad \mathbf{q}^{(3)}=\mathbf{e}^{(3)}
$$

whose magnitudes are

$$
q^{(1)}=\frac{1}{\sqrt{2}}(1-i) e^{i \phi}, \quad q^{(2)}=\frac{1}{\sqrt{2}}(1+i) e^{-i \phi}, \quad q^{(3)}=1 .
$$

The components of the tangent space and base manifold are interrelated by the tetrad (or vielbein) matrix, whose space-like components form a $3 \times 3$ invertible matrix

$$
\left[\begin{array}{l}
q^{(1)} \\
q^{(2)} \\
q^{(3)}
\end{array}\right]=\left[\begin{array}{lll}
q_{1}^{(1)} & q_{2}^{(1)} & q_{3}^{(1)} \\
q_{1}^{(2)} & q_{2}^{(2)} & q_{3}^{(2)} \\
q_{1}^{(3)} & q_{2}^{(3)} & q_{3}^{(3)}
\end{array}\right]\left[\begin{array}{l}
q^{1} \\
q^{2} \\
q^{3}
\end{array}\right] .
$$

This expression means that the coordinate system defined by the metric vectors $\mathbf{q}^{(1)}, \mathbf{q}^{(2)}, \mathbf{q}^{(3)}$ is rotating and translating with respect to the coordinate system defined by the metric vectors $\mathbf{q}^{1}, \mathbf{q}^{2}, \mathbf{q}^{3}$.

Let the components of the tetrad matrix be

$$
q_{\mu}^{a}:=q_{1}^{(1)}, q_{2}^{(1)} \ldots
$$

then these define the $O(3)$ electromagnetic field $[1,2,5-10]$ or potential:

$$
A_{\mu}^{a}:=A_{1}^{(1)}, A_{2}^{(1)}, \ldots
$$

through the Evans wave equation [2], which is an eigenequation with the tetrad

$$
A_{\mu}^{a}=A^{(0)} q_{\mu}^{a}
$$

as the eigenfunction.

The inverse of the tetrad matrix is defined by [12]

$$
A_{a}^{\mu}:=\left(A_{\mu}^{a}\right)^{-1}=\operatorname{adj} A_{\mu}^{a} /\left|A_{\mu}^{a}\right|,
$$

where $\operatorname{adj} A_{\mu}^{a}$ is the adjoint and $\left|A_{\mu}^{a}\right|$ the determinant of the tetrad matrix. (The Evans equations are obtained from the determinant $\left|A_{\mu}^{a}\right|$ 
using the appropriate Lagrangian and variational methods $[3,13])$. The adjoint matrix is the matrix of cofactors:

$$
\frac{\operatorname{adj} A_{\mu}^{a}}{A^{(0)}}=\left[\begin{array}{l}
\left(q_{2}^{(2)} q_{3}^{(3)}-q_{3}^{(2)} q_{3}^{(3)}\right)\left(q_{1}^{(2)} q_{3}^{(3)}-q_{3}^{(2)} q_{1}^{(3)}\right)\left(q_{1}^{(2)} q_{2}^{(3)}-q_{2}^{(2)} q_{1}^{(3)}\right) \\
\left(q_{2}^{(1)} q_{3}^{(3)}-q_{3}^{(1)} q_{2}^{(3)}\right)\left(q_{1}^{(1)} q_{3}^{(3)}-q_{3}^{(1)} q_{1}^{(3)}\right)\left(q_{1}^{(1)} q_{2}^{(3)}-q_{2}^{(1)} q_{1}^{(3)}\right) \\
\left(q_{2}^{(1)} q_{3}^{(2)}-q_{3}^{(1)} q_{2}^{(2)}\right)\left(q_{2}^{(1)} q_{3}^{(2)}-q_{3}^{(1)} q_{1}^{(2)}\right)\left(q_{1}^{(1)} q_{2}^{(2)}-q_{2}^{(1)} q_{1}^{(2)}\right)
\end{array}\right]
$$

The cofactors can be expressed in terms of the elements of the matrix $q_{\mu}^{a}$ by using the $O(3)$ cyclic relation that defines the orthonormal, Euclidean, tangent space $a$ :

$$
e_{2}^{(2)} e_{3}^{(3)}-e_{3}^{(2)} e_{2}^{(3)}=-i e_{1}^{(1) *}=-i e_{1}^{(2)}, \quad \text { etc. }
$$

Thus,

$$
q_{2}^{(2)} q_{3}^{(3)}-q_{3}^{(2)} q_{2}^{(3)}=-i q_{1}^{(2)}, \quad \text { etc, }
$$

and the adjoint matrix becomes

$$
\operatorname{adj} A_{\mu}^{a}=-i A^{(0)}\left[\begin{array}{ccc}
q_{1}^{(2)} & q_{1}^{(1)} & q_{1}^{(3)} \\
q_{2}^{(2)} & q_{2}^{(1)} & q_{2}^{(3)} \\
q_{3}^{(2)} & q_{3}^{(1)} & q_{3}^{(3)}
\end{array}\right]
$$

The determinant $\left|A_{\mu}^{a}\right|$ is defined by

$$
A \operatorname{adj} A=|A| I A^{(0)}, \quad\left|A_{\mu}^{a}\right|=-i A^{(0)} .
$$

The inverse matrix is therefore

$$
A_{a}^{\mu}=A^{(0)}\left[\begin{array}{ccc}
q_{1}^{(2)} & q_{1}^{(1)} & q_{1}^{(3)} \\
q_{2}^{(2)} & q_{2}^{(1)} & q_{2}^{(3)} \\
q_{3}^{(2)} & q_{3}^{(1)} & q_{3}^{(3)}
\end{array}\right]
$$

and we arrive at the equations

$$
A^{(0)}\left[\begin{array}{l}
q^{(1)} \\
q^{(2)} \\
q^{(3)}
\end{array}\right]=A_{\mu}^{a}\left[\begin{array}{l}
q^{1} \\
q^{2} \\
q^{3}
\end{array}\right], \quad A^{(0)}\left[\begin{array}{l}
q^{1} \\
q^{2} \\
q^{3}
\end{array}\right]=A_{a}^{\mu}\left[\begin{array}{l}
q^{(1)} \\
q^{(2)} \\
q^{(3)}
\end{array}\right] .
$$


In the notation of differential geometry [3], these two equations are written as

$$
A^{(0)} q^{a}=A_{\mu}^{a} q^{\mu}, \quad A^{(0)} q^{\mu}=A_{a}^{\mu} q^{a}
$$

and define the tetrad $A_{\mu}^{a}$ and the inverse tetrad $A_{a}^{\mu}$. The tetrad and its inverse define the FIELDS in grand unified field theory. In other words, all four fields currently thought to exist are defined by the way in which the tangent space is related to the base manifold. The interrelation of fields is also defined by the tetrad. In the presence of gravitation, for example, the base manifold becomes curved, and the tetrad defining the electromagnetic field in Eq. (12) changes, so the electromagnetic field changes due to the presence of gravitation.

These considerations can be extended to the four dimensions of spacetime by interrelating the two basis four-vectors

$$
\begin{aligned}
& q^{a}:=\left(q^{(0)}, q^{(1)}, q^{(2)}, q^{(3)}\right), \\
& q^{\mu}:=\left(q^{0}, q^{1}, q^{2}, q^{3}\right)
\end{aligned}
$$

in which the tetrad is defined by the four by four invertible matrix

$$
A^{(0)}\left[\begin{array}{l}
q^{(0)} \\
q^{(1)} \\
q^{(2)} \\
q^{(3)}
\end{array}\right]=A_{\mu}^{a}\left[\begin{array}{l}
q^{0} \\
q^{1} \\
q^{2} \\
q^{3}
\end{array}\right], \quad A^{(0)}\left[\begin{array}{l}
q^{0} \\
q^{1} \\
q^{2} \\
q^{3}
\end{array}\right]=A_{a}^{\mu}\left[\begin{array}{l}
q^{(0)} \\
q^{(1)} \\
q^{(2)} \\
q^{(3)}
\end{array}\right]
$$

whose adjoint (matrix of cofactors) reads

$$
\operatorname{adj} A_{\mu}^{a}=-i\left[\begin{array}{cccc}
q_{0}^{(0)} & q_{0}^{(2)} & q_{0}^{(1)} & q_{0}^{(3)} \\
q_{1}^{(0)} & q_{1}^{(2)} & q_{1}^{(1)} & q_{1}^{(3)} \\
q_{2}^{(0)} & q_{2}^{(2)} & q_{2}^{(1)} & q_{2}^{(3)} \\
q_{3}^{(0)} & q_{3}^{(2)} & q_{3}^{(1)} & q_{3}^{(3)}
\end{array}\right]
$$

and whose determinant is

$$
\left|A_{\mu}^{a}\right|=-i
$$

The inverse matrix (or inverse tetrad) is therefore defined by

$$
A_{\mu}^{a} A_{a}^{\nu}=\delta_{\mu}^{\nu}
$$

We have gone through this exercise in detail to illustrate the meaning of the tetrad in $O(3)$ electrodynamics. It is essentially the matrix that 
inter-relates two frames of reference: that of the base manifold and the orthonormal tangent space. The tetrad is the eigenfunction of the Evans wave equation [2], which for $O(3)$ electrodynamics becomes the eigenequation

$$
(\square+k T) A_{\mu}^{a}=0,
$$

where $k$ is the gravitational constant and $T$ the contracted energymomentum tensor [2]. The wave equation (27) is a direct consequence of the tetrad postulate [2,3], which for $O(3)$ electrodynamics is

$$
D_{\nu} A_{\mu}^{a}=0
$$

representing a cyclic relation [2] between tetrad components (components of the $O(3)$ electromagnetic field). The tetrad is centrally important to the theory of general relativity expressed as a theory of differential geometry $[3,13]$ and is used to derive the Maurer-Cartan structure relations [3] between differential forms: the Riemann and torsion forms, valid for all types of connection. By recognizing that the tetrad is the fundamental eigenfunction in the Evans wave equation [2], it follows (Sec. 3) that the Maurer-Cartan structure relations become field/matter relations of grand unified field theory, i.e., of all the known force fields and matter waves in nature. The wave equation [2] is the quantized version of the classical field/matter equation introduced in Ref. [1]:

$$
R_{\mu}-\frac{1}{2} R q_{\mu}=k T_{\mu}
$$

Equation (29) is written in terms of four-vectors in a base manifold that is in general a non-Euclidean spacetime. The field tensor in Eq. (29) is the four-vector

$$
G_{\mu}=R_{\mu}-\frac{1}{2} R q_{\mu}
$$

where $R_{\mu}$ is the Ricci four-vector, $R$ the scalar curvature, $q_{\mu}$ the metric four-vector, $k$ is the Einstein constant, and $T_{\mu}$ the canonical energy-momentum four-vector. In the language of differential geometry [2,3,13], Eq. (29) becomes a relation between the corresponding differential one-forms. In tetrad notation, Eq. (29) is

$$
G_{\mu}^{a}=k T_{\mu}^{a}
$$

In differential geometry, the equation becomes

$$
G^{a}=k T^{a}
$$

where the index $\mu$ is implied [3]. The wave equation is differential geometry is the eigenequation with the one form (tetrad) $q^{a}$ as eigenfunction:

$$
(\square+k T) q^{a}=0 .
$$


The $O(3)$ electromagnetic gauge field is then the two-form [2]

$$
G^{c}=G^{(0)} q^{a} \wedge q^{b}
$$

where the wedge produce is a wedge product between one-forms [3]:

$$
\left(q^{a} \wedge q^{b}\right)_{\mu \nu}:=q_{\mu}^{a} q_{\nu}^{b}-q_{\nu}^{a} q_{\mu}^{b}
$$

A central result of this theory is that electrodynamics is a gauge field (Eq. (34)) whose internal index $c$ is the tangent index of the base manifold in general relativity. The tangent space is an orthonormal Euclidean space whose unit vectors form an $O(3)$ symmetry cyclic relation:

$$
\mathbf{e}^{(1)} \times \mathbf{e}^{(2)}=i \mathbf{e}^{(3) *}, \quad \text { et cyclicum. }
$$

Thus, generally covariant electrodynamics in an $O(3)$ symmetry gauge field theory $[1,2,5-10]$. This is a fundamental result of general relativity and differential geometry.

\section{GUFT AS DIFFERENTIAL GEOMETRY: EVANS EQUATIONS AND THE MAURER-CARTAN STRUCTURE RELATIONS}

Equation (31) is the Evans field equation (29) for each index $a$. The quantities in Eq. (29) are defined by [1-3]

$$
R_{\mu \nu}=R_{\mu} q_{\nu}, \quad q_{\mu \nu}=q_{\mu} q_{\nu}, \quad T_{\mu \nu}=T_{\mu} q_{\nu}
$$

where $R_{\mu \nu}$ is the Ricci tensor, $q_{\mu \nu}$ the symmetric metric tensor, and $T_{\mu \nu}$ the symmetric canonical energy-momentum tensor of Einstein's general relativity [3]. The symmetric metric tensor is defined as

$$
q_{\mu \nu}=q_{\mu} q_{\nu}
$$

and $[14]$

$$
q^{\mu \nu} q_{\mu \nu}=4
$$

Considering the flat spacetime limit:

$$
q^{\mu} q_{\mu}=-2, \quad q^{\mu}=(1,1,1,1), \quad q_{\mu}=(1,-1,-1,-1) .
$$

More generally, Eq. (40) indicates that, in non-Euclidean spacetime, $q^{\mu}$ is the inverse metric if $q_{\mu}$ is the metric. The scalar curvature is

$$
R=q^{\mu \nu} R_{\mu \nu}=q^{\mu} q^{\nu} R_{\mu} q_{\nu}=-2 q^{\mu} R_{\mu} ;
$$


and multiplying Eq. (41) on both sides by $q_{\mu}$ gives

$$
R_{\mu}=\frac{1}{4} R q_{\mu}
$$

The Evans field equation (29) can therefore be developed as

$$
G_{\mu}^{a}=-\frac{1}{4} R q_{\mu}^{a}=k T_{\mu}^{a} .
$$

Similarly, the contracted energy-momentum tensor is defined by Einstein [14] as

$$
T=q^{\mu \nu} T_{\mu \nu}=-2 q^{\mu} T_{\mu}
$$

Multiplication of Eq. (44) on both sides by $q_{\mu}$ gives

$$
T_{\mu}=\frac{1}{4} T q_{\mu}
$$

Therefore, the Evans field equation becomes the classical equation

$$
R q_{\mu}^{a}=-k T q_{\mu}^{a}
$$

which is the contracted form of Einstein's field equation

$$
R=-k T
$$

multiplied on both sides by the tetrad $q_{\mu}^{a}$. The Evans wave equation $[2]$

$$
\square q_{\mu}^{a}=R q_{\mu}^{a}=-k T q_{\mu}^{a}
$$

is obtained from the tetrad postulate and is the quantized version of Eq. (47).

The classical and quantum Evans equations are equations for the fundamental field in grand unified field theory. In both cases the field is recognized as the tetrad. The tetrad represents the components indexed $\mu$ of the coordinate basis vectors in terms of the components indexed $a$ of the orthonormal basis defining the vectors o the tangent space in general relativity. In differential geometry the tetrads are also the components of the orthonormal basis one-forms in terms of the coordinate basis one forms [3].

The classical Eq. (4b) states that

$$
R+k T=0
$$

by ansatz, the basic postulate of general relativity. The quantum Eq. (48) originates in the tetrad postulate [3] of geometry, a postulate which is true for any connection, whether torsion free or not, and 
Eq. (48) gives quantum field/matter theory from general relativity. Gravitation is described by these equations when the field is the tetrad $q_{\mu}^{a}$. The other three fields are described when the field is the tetrad multiplied by an appropriate scaling factor. For example, the fundamental electromagnetic field is

$$
A_{\mu}^{a}=A^{(0)} q_{\mu}^{a},
$$

where $A^{(0)}$ is the magnitude of the electromagnetic four-potential, as described in Sec. 1.

The gauge invariant fields, or gauge fields, are defined as follows. The gravitational gauge field is the Riemann form of differential geometry, the dual of the torsion tensor:

$$
R_{a b}=\frac{R}{4} \varepsilon_{a b c d}\left[q^{c}, q^{d}\right] .
$$

The other three fields are defined by the torsion form, the antisymmetric sum or commutator of tetrads:

$$
\tau_{\mu \nu}^{c}:=\frac{R}{4}\left[q_{\mu}^{a}, q_{\nu}^{b}\right]=\frac{R}{4}\left(q_{\mu}^{a} q_{\nu}^{b}-q_{\nu}^{a} q_{\mu}^{b}\right) .
$$

The tetrad is a vector valued one form [3]. Define its exterior derivative as the vector valued two-form

$$
(d q)_{\mu \nu}^{a}:=\partial_{\mu} q_{\nu}^{a}-\partial_{\nu} q_{\mu}^{a}
$$

and its covariant exterior derivative as

$$
\begin{aligned}
(D q)_{\mu \nu}^{a} & =(d q+\omega \wedge q)_{\mu \nu}^{a} \\
& =\partial_{\mu} q_{\nu}^{a}-\partial_{\nu} q_{\mu}^{a}+\omega_{\mu b}^{a} q_{\nu}^{b}-\omega_{\nu b}^{a} q_{\mu}^{b},
\end{aligned}
$$

where $\omega$ is the spin connection [3].

The first Maurer-Cartan structure relation [3] is then

$$
\tau^{c}=D q^{c},
$$

and the second Maurer-Cartan structure relation is

$$
R_{b}^{a}=D \omega_{b}^{a} .
$$

We therefore arrive at the following equations which combine the two Evans equations with the two Maurer-Cartan structure relations to define the gauge invariant fields in grand unified field theory:

$$
R_{b}^{a}=\varepsilon_{b c}^{a} T^{c},
$$


where $T^{c}$ is the torsion form,

$$
\tau^{c}=\frac{R}{4}\left[q^{a}, q^{b}\right]=D q^{c} .
$$

The gravitational field is described by the Riemann form and the other three fields by the torsion form multiplied by the scale factor $G^{(0)}$ :

$$
G^{c}=G^{(0)} \tau^{c}
$$

In $O(3)$ electrodynamics the scale factor is the primordial magnetic flux density (in units of tesla, or webers per square meter):

$$
B^{(0)}=(1 / 4) G^{(0)} R \text {. }
$$

Equations (57) and (58) also define the spin connection in terms of the tetrad for all four fields.

Differential geometry [3] also gives the following identities:

$$
\begin{gathered}
D R_{b}^{a}:=0, \\
D^{*} \tau^{c}:=0, \\
D \tau^{c}=R_{b}^{c} \wedge q^{b},
\end{gathered}
$$

which can be used to interrelate the four gauge fields. In the following section, several novel results are given by interrelating the gravitational and electromagnetic fields. Equation (61) is the Bianchi identity, and Eq. (62) and (63) are the homogeneous and inhomogeneous gauge field equations. In $O(3)$ electrodynamics Eq. (63) shows that the current term is derived from the Riemann form, and therefore from the scalar curvature $R$ multiplied by the anti-commutator of tetrads.

This fundamental result of differential geometry implies that electromagnetic energy can be transmitted from a source to a receiver by scalar curvature $R$ and that electromagnetic energy is available in non-Euclidean spacetime.

Experimental evidence supporting ths result might be found in devices such as the patented motionless electromagnetic generator [15] and Sweet's device [16]. All fields in nature are fundamentally dependent upon, and originate in, scalar curvature $R$.

\section{INTER-RELATION OF FIELDS: THE POISSON EQUATION AND OTHER RESULTS}

The Evans wave equation [2] is obtained by covariant differentiation of the tetrad postulate (28) and reads

$$
D^{\rho} D_{\rho} q_{\mu}^{a}=(\square+k T) q_{\mu}^{a}=(\square-R) q_{\mu}^{a}=0,
$$


where $\square$ is the d'Alembertian in Euclidean spacetime (the spacetime of special relativity). The scalar curvature in this equation is [2]

$$
R=-D^{\mu} \Gamma_{\mu \rho}^{\rho}=-\left(\partial^{\mu} \Gamma_{\mu \rho}^{\rho}+\Gamma_{\lambda}^{\mu \rho} \Gamma_{\mu \rho}^{\lambda}\right)
$$

We therefore deduce that

$$
\partial^{\mu} \Gamma_{\mu \rho}^{\rho}+\Gamma_{\lambda}^{\mu \rho} \Gamma_{\mu \rho}^{\lambda}=k T .
$$

Both Eqs. (64) and (66) give Poisson's equation in the appropriate approximation. The equivalence principle states that the laws of physics in small enough regions of spacetime reduce to the equations of special relativity $[3,4]$. In special relativity there exist equations such as

$$
\begin{gathered}
\left(\square+\kappa_{0}^{2}\right) \phi=0, \\
\left(\square+\kappa_{0}^{2}\right) \psi=0, \\
\left(\square+\kappa_{0}^{2}\right) A_{\mu}=0,
\end{gathered}
$$

where $\lambda_{0}$ is the Compton wavelength of a particle:

$$
\lambda_{0}=1 / \kappa_{0}=\hbar / m c .
$$

Here $m$ is the particle mass, $\hbar$ is the Dirac constant $h / 2 \pi$, and $c$ the speed of light in vacuum. Equation (67) is the Klein-Gordon equation, in which $\phi$ is the scalar field; Eq. (68) is the Dirac equation, in which $\psi$ is the four spinor; and Eq. (69) is the Proca equation, in which $A_{\mu}$ is the electromagnetic wave function, conventionally a four-vector of the Maxwell Heaviside theory [4]. (In generally covariant electrodynamics $A_{\mu}^{a}$ is a tetrad, as we have already argued.) In order to reduce Eq. (64) to Eqs. (67), (68), and (69), we define the latter as limiting forms of the Evans wave equation of general relativity, Eq. (64). The limiting forms are obtained when the rest curvature $R_{0}$ is defined by

$$
\left|R_{0}\right|=\kappa_{0}^{2}=k T_{0},
$$

where

$$
T_{0}=m / V=q^{0} q^{0} T_{00}, \quad q^{0}:=1 .
$$

The rest curvature is therefore the inverse square of the Compton wavelength for any particle, including the photon:

$$
\left|R_{0}\right|=m^{2} c^{2} / \hbar^{2} .
$$

The rest curvature is defined by the rest energy:

$$
E_{0}=m c^{2}=\hbar c \sqrt{\left|R_{0}\right|},
$$


where

$$
\kappa_{0}=\sqrt{\left|R_{0}\right|} .
$$

More generally, the quantum of energy for any particle (not only the photon) is given by

$$
E=\hbar c \sqrt{|R|}
$$

where

$$
|R|=D^{\mu} \Gamma_{\mu \rho}^{\rho}
$$

Equation (76) is therefore a generalization of the Planck postulate for the photon in special relativity to all particles in general relativity.

On writing

$$
E=\hbar \omega=\hbar c \sqrt{|R|},
$$

it becomes clear the the quantum $\hbar \omega$ is electromagnetic energy, e.g., is available from scalar curvature $R$, i.e., from any kind of connection $\Gamma_{\mu \rho}^{\rho}$, and for any sources of $R$. Equation (63) is a statement of this result in classical electrodynamics.

It is now demonstrated that the well-known Poisson equation for both Newtonian dynamics and for electro-statics can be obtained self consistently both from Eq. (64) and Eq. (66). Starting from Eq. (64), the Poisson equation is obtained from the component $q_{0}^{0}$ :

$$
\left(\square+\frac{k m}{V}\right) q_{0}^{0}=0,
$$

in which $T=m / V$ is the mass density and $V$ is a volume. The rest mass density $m / V_{0}$, where $V_{0}$ is the rest volume, can be regarded as the zero-point energy of the Evans wave equation of generally covariant quantum field theory. In the weak field or Newtonian limit [1-3], Eq. (79) becomes

$$
\square q_{0}^{0}=-\frac{k m}{V} q_{0}^{0} \sim-\frac{k m}{V},
$$

because in this limit

$$
q_{0}^{0}=1+\eta_{0}^{0},
$$

where $\eta_{0}^{0}$ is a small perturbation of the tetrad. In the Newtonian limit, $q_{0}^{0}$ is a quasi-static, so Eq. (80) reduces to

$$
\nabla^{2} \Phi=4 \pi G \rho
$$

which is the Poisson equation of Newtonian dynamics [3,17] provided that

$$
\Phi:=\frac{1}{2} c^{2} q_{0}^{0}
$$


where $G$ is Newton's gravitational constant and $\rho$ the mass density $m / V$.

In order to derive Eq. (82) from Eq. (66), it is assumed that in the weak-field limit the term quadratic in the connection can be neglected and the Eq. (72) applies, so that the relevant component of the metric vector $q_{\mu}$ in the non-Euclidean base manifold is $q_{0}$. In the Newtonian limit

$$
q^{0}=1+\eta^{0}
$$

where $\eta^{0} \ll q^{0}$. The metric vector is assumed to be metric compatible $[2,3]$, and the equation of metric compatibility for $q^{\mu}$ is

$$
D_{\nu} q^{\mu}=\partial_{\nu} q^{\mu}+\Gamma_{\nu \lambda}^{\mu} q^{\lambda}=0 .
$$

The relevant index to consider is $\lambda=0$, and thus

$$
\partial_{\nu} q^{\mu}+\Gamma_{\nu 0}^{\mu} q^{0}=0
$$

Multiplying by $q_{0}$ and using $q_{0} q^{0}=1$, one gets

$$
\Gamma_{\nu 0}^{\mu}=-q_{0} \partial_{\nu} q^{\mu} \sim-\partial_{\nu} q^{\mu}
$$

where Eq. (84) has been used. The relevant index in Eq. (87) is $\mu=0$, giving

$$
\Gamma_{\nu 0}^{0}=-\partial_{\nu} q^{0}
$$

so that Eq. (66) becomes

$$
\partial^{\mu} \Gamma_{\mu 0}^{0}=-\square q^{0}=\left(8 \pi / c^{2}\right) G \rho .
$$

In the Newtonian limit the field is quasi-static, so Eq. (89) reduces to the Poisson Eq. (82), provided that

$$
\Phi:=\frac{1}{2} c^{2} q^{0}
$$

These two derivations of the Poisson equation in the Newtonian limit show that Eq. (64) is consistent Eq. (66) and that there exists a rest curvature corresponding to rest energy in special relativity. The rest curvature produces energy which is convertible into any other form of energy, including electromagnetic energy; and, in grand unified field theory, electromagnetic energy originates in general from curvature $R$. Equations (67) and (69) are limiting forms of Eq. (64) when the curvature is the rest curvature. Therefore the scalar field, the spinor field, and the conventional electromagnetic field of Maxwell Heaviside field theory are all limiting forms of the tetrad.

The Poisson equation in electrostatics is obtained, using the same approximations, from the Evans wave equation (64) when its 
eigenfunction is the electromagnetic tetrad field defined by Eq. (50). The Poisson equation for electrostatics is therefore a limiting form of the equation

$$
(\square+k T) A_{\mu}^{a}=(\square-R) A_{\mu}^{a}=0
$$

in general relativity and grand unified field theory; it is

$$
\nabla^{2}\left(A^{(0)} \Phi\right)=4 \pi G\left(A^{(0)} \rho\right)
$$

The scalar potential in electrostatics is therefore

$$
\phi=(1 / c) A^{(0)} \Phi
$$

and the Poisson equation in electrostatics is the Schrödinger-type eigenequation

$$
\nabla^{2} \phi=\left(4 \pi G \rho / c^{2}\right) \phi .
$$

The units of $\phi$ are $\mathrm{JC}^{-1}$ and the units of $A^{(0)}$ are $\mathrm{JsC}^{-1} \mathrm{~m}^{-1}$, and so

$$
\phi=c A^{(0)} .
$$

The Poisson equation is therefore an eigenequation in the electrostatic potential $\phi$, and this result can be thought of as the quantization of charge because the quantity $\varepsilon_{0} \phi$ where $\varepsilon_{0}$ is the vacuum permittivity (S.I. Units) has the units of $\mathrm{C} \mathrm{m}^{-1}$. Therefore if electrostatics is considered to be an approximation of generally covariant grand unified field theory, we obtain the equation of quantization of charge:

$$
\nabla^{2} \psi_{e}=\left(4 \pi G \rho / c^{2}\right) \psi_{e}
$$

where $\psi_{e}=\varepsilon_{0} \phi$ and

$$
\varepsilon_{0}=8.854188 \times 10^{-12} \mathrm{~J}^{-1} \mathrm{C}^{2} \mathrm{~m}^{-1}, \quad G=6.6726 \times 10^{-11} \mathrm{Nm}^{2} \mathrm{~kg}^{-2} .
$$

The Poisson equation in S.I. units in electrostatics is usually written as

$$
\nabla^{2} \phi=-\rho_{e} / \varepsilon_{0}
$$

where $\rho_{e}$ is the charge density in $\mathrm{C} \mathrm{m}^{-3}$. The minus sign in Eq. (97) is a matter of convention [18]; it is chosen so that the electric field strength is defined by

$$
\mathbf{E}:=-\nabla \phi,
$$

i.e., points towards a decrease in potential. The factor $4 \pi$ is conventionally divided out of the Poisson equation for electrostatics [18] as a matter of convenience in the S.I. system of units. Therefore the Poisson 
equation for electrostatics can always be written in the same form as that for Newtonian dynamics:

$$
\nabla^{2} \phi=\left(4 \pi / \varepsilon_{0}\right) \rho_{e}
$$

where the charge density $\rho_{e}$ and mass density $\rho$ are, respectively,

$$
\rho_{e}=\int e d V=\left(\varepsilon_{0} G / c^{2}\right) \phi^{(0)} \rho, \quad \rho=\int m d V .
$$

Here $e$ is the fundamental charge (defined as the charge on the proton, or the modulus of the charge on the electron) and $m$ the mass of the electron:

$$
e=1.60219 \times 10^{-19} \mathrm{C}, \quad m=9.10953 \times 10^{-31} \mathrm{~kg} .
$$

Comparison of Eqs. (94) and (99) yields

$$
\rho_{e}=\left(\varepsilon_{0} G / c^{2}\right) \phi^{(0)} \rho,
$$

giving the fundamental ratio of charge $e$ to mass $m$ in terms of the fundamental electrostatic potential $\phi^{(0)}$ :

$$
e=\left(\varepsilon_{0} G / c^{2}\right) \phi^{(0)} m .
$$

Application of (103) to the electron gives

$$
\phi^{(0)}(\text { one electron })=\frac{c^{2} e}{\varepsilon_{0} G m} \mathrm{JC}^{-1},
$$

which is a fundamental constant furnishing the number of joules available from the ratio $e / m$ for one electron in Eq. (104),

$$
\begin{aligned}
& c=2.997925 \times 10^{8} \mathrm{~m} \mathrm{~s}^{-1}, \\
& e=1.60219 \times 10^{-19} \mathrm{C}, \\
& \varepsilon_{0}=8.854188 \times 10^{-12} \mathrm{~J}^{-1} \mathrm{C}^{2} \mathrm{~m}^{-1}, \\
& G=6.6726 \times 10^{-11} \mathrm{Nm}^{2} \mathrm{~kg}^{-2}, \\
& m=9.10953 \times 10^{-31} \mathrm{~kg},
\end{aligned}
$$

and so

$$
\begin{aligned}
& \phi^{(0)}(\text { one electron })=2.6726 \times 10^{49} \mathrm{~J} \mathrm{C}^{-1}, \\
& A^{(0)}(\text { one electron })=8.92473 \times 10^{40} \mathrm{~J} \mathrm{C}^{-1}, \mathrm{~s} \mathrm{~m}^{-1} .
\end{aligned}
$$


It now becomes possible to interrelate the fundamental equations of Newtonian dynamics and electrostatics by using Eqs. (102) and (103) to interrelate terms in the following well-known equations:

$$
\begin{gathered}
\mathbf{g}=-\boldsymbol{\nabla} \Phi, \quad \mathbf{E}=-\boldsymbol{\nabla} \phi ; \\
\mathbf{F}=-\left(G m_{1} m_{2} / r^{2}\right) \mathbf{k}=m_{1} \mathbf{g}, \quad \mathbf{F}=-\left(e_{1} e_{2} / \varepsilon_{0} r^{2}\right) \mathbf{k}=e_{1} \mathbf{E}, \\
\boldsymbol{\nabla}=\left(G m_{2} / r^{2}\right) \mathbf{k}, \quad \boldsymbol{\nabla} \phi=\left(e_{2} / \varepsilon_{0} r^{2}\right) \mathbf{k}
\end{gathered}
$$

in which $\mathbf{g}$ is the gravitational acceleration, $r$ the distance between two masses $m_{1}$ and $m_{2}$ or two charges $e_{1}$ and $e_{2}$, and $\mathbf{F}$ is the force between the two masses of the two charges.

All these well-known equations originate in the wave equation (64) of generally covariant grand unified field theory. Both the gravitational and the electrostatic fields originate in the tetrad when approximated in the weak-field limit in which the tetrad is a perturbation of Euclidean spacetime and in which the field is quasistatic. equation

All forms of energy are inter-convertible, and it follows from the

$$
R=-k T
$$

that all forms of curvature $R$ are interconvertible. Newtonian dynamics is the weak-field limit of the wave equation (64), and electrostatics is the weak-field limit of the same equation multiplied by $A^{(0)}$. It follows that, given a charge $e$, there is an equivalent amount of mass $m$. If the force in Newton's between two masses a distance $r$ apart is numerically the same as that between two charges the same distance $r$ apart, then

$$
e^{2}=\varepsilon_{0} G m^{2} .
$$

From this equation,

$$
e= \pm 2.430647 \times 10^{-11} \mathrm{~m}, \quad m= \pm 4.11413 \times 10^{10}|e|,
$$

which shows that if there is only one sign of mass (as observed experimentally). Charge can be thought to originate in "symmetry breaking" of mass into two different signs, and mass originates in curvature of non-Euclidean spacetime, something which can be loosely described as a "symmetry breaking of the vacuum." Therefore there can be neither charge nor mass in Euclidean spacetime. apart, is

The force between two charges of one coulomb each, one meter

$$
1 / \varepsilon_{0}=1.12941 \times 10^{11} \mathrm{~N}
$$

and the force between two masses of one kilogram each, one meter apart, is

$$
G=6.6726 \times 10^{-11} \mathrm{~N} .
$$


The electrostatic force is therefore

$$
1 / \varepsilon_{0} G=1.69261 \times 10^{21}
$$

times greater than the gravitational force, which is why the gravitational force is conventionally referred to as "weak" in comparison with the electrostatic force in the laboratory. However, both forces originate in rest curvature $R_{0}$. From Eq. (102), the charge density is given by

$$
\rho_{e}=-\left(\varepsilon_{0} \phi^{(0)} / 8 \pi\right) R_{0},
$$

and the mass density is

$$
\rho=-R / k
$$

The Einstein constant is

$$
k=8 \pi G / c^{2}=1.86595 \times 10^{-26} \mathrm{~N} \mathrm{~s}^{2} \mathrm{~kg}^{-2},
$$

and thus

$$
\rho_{e}=-3.5229 \times 10^{-13} \phi^{(0)} R_{0}, \quad \rho=-5.35920 \times 10^{25} R_{0} .
$$

If the fundamental potential (106) is used in Eq. (119), the following results are obtained:

$$
\rho_{e}=-9.41530 \times 10^{36} R_{0}, \quad \rho=-5.35920 \times 10^{25} R_{0} .
$$

The charge obtainable from a given curvature $R$ in a given volume is about twelve orders of magnitude greater than the mass obtainable from the same curvature $R$ for the same volume.

From Eq. (79) it is possible to define the rest volume of a particle:

$$
V_{0}=\frac{8 \pi G \hbar^{2}}{m c^{4}}=\frac{8 \pi \lambda_{p}^{2}}{\sqrt{\left|R_{0}\right|}}
$$

where

$$
\lambda_{c}=1 / \sqrt{\left|R_{0}\right|}=\hbar / m c
$$

is the Compton wavelength, and where $\lambda_{p}$ denotes the Planck length

$$
\lambda_{p}=\sqrt{G \hbar / c^{3}} .
$$

For the electron,

$$
\lambda_{c}=2.42631 \times 10^{-12} \mathrm{~m}, \quad \lambda_{p}=4.05087 \times 10^{-35} \mathrm{~m},
$$

and the rest volume is therefore

$$
V_{0}=8 \pi \lambda_{c} \lambda_{p}^{2}=1.00065 \times 10^{-79} \mathrm{~m}^{3} .
$$


Equation (122) interrelates wave-particle dualism and general relativity.

The Planck and Compton wavelengths can be expressed in terms of the rest curvature $R_{0}$ as follows:

$$
\lambda_{c}=\left(c^{2} / G m\right) \lambda_{p}^{2}=1 / \sqrt{\left|R_{0}\right|} .
$$

The classical radius of the electron $\left(\lambda_{e}\right)$ can also be expressed in terms of rest curvature $R_{0}$ :

$$
\lambda_{e}=\alpha \lambda_{0}, \quad \lambda_{0}=1 / \sqrt{\left|R_{0}\right|},
$$

where

$$
\alpha=e^{2} / 4 \pi \varepsilon_{0} \hbar c
$$

is the fine structure constant of electrodynamics:

$$
\alpha=7.297351 \times 10^{-3} .
$$

Equation (127) derives quantum electrodynamics from generally covariant grand unified field theory.

The rest wave-number

$$
\kappa_{0}=1 / \lambda_{0}=\sqrt{\left|R_{0}\right|}
$$

is fundamental to the interrelation of general relativity with quantum mechanics and classical and quantum electrodynamics. The KleinGordon, Dirac, and Proca equations can each be written in terms of the rest wavenumber, which is the inverse of the Compton wavelength:

$$
\begin{gathered}
\left(\square-R_{0}\right) \phi=0, \\
\left(\square-R_{0}\right) \psi=0, \\
\left(\square-R_{0}\right) A_{\mu}=0 .
\end{gathered}
$$

The Evans wave equation shows that

$$
D^{\mu} \Gamma_{\mu \rho}^{\rho} \rightarrow-R_{0}
$$

in the limit of special relativity.

The equation of quantization of mass is

$$
m=\hbar \kappa_{0} / c:=\hbar \omega_{0} / c^{2}
$$

The ratio of charge to mass can always be expressed as

$$
e^{2} / m^{2}=\zeta \varepsilon_{0} G,
$$


where $\zeta$ is a dimensionless coefficient. Equation (136) is the result of the Newton and Coulomb inverse square laws, which we have derived from the Evans equation in the weak-field limit. Therefore the Newton and Coulomb inverse square laws become the same. Using Eq. (135) in Eq. (136),

$$
e= \pm \hbar \kappa_{0} \sqrt{\zeta \varepsilon_{0} G / c^{2}}
$$

Comparing Eqs. (136) and (102), one gets

$$
\zeta=\left(e / m c^{2}\right) \phi
$$

and thus Eq. (137) is the charge quantization equation.

All these results emerge from the Evans wave equation in the weak-field approximation.

Equation (135) can be generalized to

$$
m=\hbar \kappa_{0} / c=\hbar \sqrt{|R|} / c,
$$

i.e., to

$$
E=\hbar c \sqrt{|R|}=\hbar c \kappa=\hbar \omega,
$$

which is Eq. (76) for the quantization of energy in terms of the wavenumber $\sqrt{|R|}$. Similarly, Eq. (139) can be generalized to

$$
e= \pm(\hbar / c) \sqrt{\zeta \varepsilon_{0} G|R|}
$$

Equations (140) and (141) are generally covariant equations for the quantization of charge and mass in terms of the wave number $\sqrt{|R|}$, where $R+k T=0$. They show that mass and charge are quantized in terms of the fundamental wavenumbers:

$$
\kappa=\sqrt{|R|}
$$

\section{DISCUSSION}

It has been shown that all force and matter fields in nature are determined and interrelated by the two Evans equations [1,2] combined with the Maurer-Cartan structure relations of differential geometry [3]. The homogeneous and inhomogeneous gauge field equations then follow from geometrical considerations, and interrelate the torsion and Riemann forms. The gauge field equations show how the force and matter fields are interrelated. Some consequences of this deduction are worked out in Sec. 4, and there are many other consequences yet to be inferred and tested experimentally. One of the major predictions of 
the Evans equations, the existence of higher symmetry electrodynamics, has been extensively developed and tested experimentally [5-10]; for example, the fundamental $\mathbf{B}^{(3)}$ field of generally covariant electrodynamics has been observed experimentally in the inverse Faraday effect, in the phase factor of physical optics and interferometry, and in several other ways. Another major prediction of the Evans equations is the availability of electromagnetic energy from non-Euclidean spacetime. This prediction appears to be verified experimentally in devices such as the patented electromagnetic generator [15] and in Sweet's device [16]. Development of such devices could lead to the general availability of electromagnetic energy, energy which originates in the wavenumber $\sqrt{|R|}$. The Evans equations show that the acquisition of such energy does not violate Noether's Theorem. Energy is obtained from $k T$ in a source situated anywhere in the universe and is transmitted to the receiver by the scalar curvature $R=-k T$ of non-Euclidean spacetime, sometimes referred to loosely as "the vacuum." The popular phrase "energy from the vacuum" [15] does not imply "energy from nothing at all."

The most important aspect of a grand unified field theory is its ability to interrelate fields, and in Sec. 4 we have illustrated this by interrelating, and thus identifying, charge and mass through the fundamental potential. Since all forms of scalar curvature are interconvertible, electromagnetic energy is obtainable from any type of scalar curvature anywhere in the universe, given the existence of the primordial fluxon $\hbar / e$ anywhere in the universe. Therefore electromagnetic energy from non-Euclidean spacetime is available to the Earthbound engineer in usable form and originates in the curvatures inherent in the rest of the universe. The Evans field and wave equations describe how the energy propagates from source to Earth-bound engineer in usable form, and originates in the curvatures inherent in the rest of the universe. The Evans field and wave equations describe how the energy propagates from source to Earth-bound observer. It has been shown in Sec. 4 that the charge generated by a given curvature $R$ is about twelve orders of magnitude greater than the mass generated by the same curvature $R$ in a given volume. This means that the charge and concomitant electromagnetic energy available from curvature induced by mass is amplified by about twelve orders of magnitude. Loosely writing, a small amount of mass results in a very large amount of charge, and this augurs well for the design of devices which can trap and use this electromagnetic energy.

In general the Evans field and wave equations are nonlinear in the connection and require numerical methods for solution, but in Sec. 4 it has been shown that approximate analytical methods of solution can lead to powerful results on the interrelation of fields. The field is the tetrad, and the influence of one type of field (e.g., gravitational), on another (e.g., electromagnetic) is measured through changes in tetrad 
components as discussed in Sec. 2. It is important to develop the interrelation of the $O(3)$ electromagnetic field with the weak field and the gravitational field with the strong field by developing and interrelating the bases used for the tangent space and fiber bundle space. This will be the subject of further work. It is already clear however that the four fields thought to exist in nature have a common origin, the scalar curvature $R$.

Acknowledgments. Many formative discussions are gratefully acknowledged with the Fellows and Emeriti of the AIAS, and funding from the Ted Annis Foundation and Craddock, Inc. is gratefully acknowledged.

\section{REFERENCES}

1. M. W. Evans, Found. Phys. Lett. 16, 369 (2003).

2. M. W. Evans, Found. Phys. Lett., in press (2003); www.aias.us

3. S. M. Carroll, Lecture Notes in General Relativity (University of California, Santa Barbara, graduate course on arXiv:grqc/9712019 v1 3 Dec., 1997).

4. L. H. Ryder, Quantum Field Theory (Cambridge University Press, Cambridge, 1987).

5. M. W. Evans, J.-P. Vigier, et al. The Enigmatic Photon (Kluwer Academic, Dordrecht, 1994 to 2002, hardback and paperback; Japanese translation in preparation, 2003; www.aias.us).

6. M. W. Evans and L. B. Crowell, Classical and Quantum Electrodynamics and the $\mathbf{B}^{(3)}$ Field (World Scientific, Singapore, 2001); www.aias.us

7. M. W. Evans, ed., Modern Non-linear Optics, in I. Prigogine and S. A. Rice, series eds., Advances in Chemical Physics, Vol. 119 (Wiley-Interscience, New York, 2001); www.aias.us; see also P. K. Anastasowski and D. Hamilton, Vol. 119 (3).

8. D. J. Clements and M. W. Evans, Found. Phys. Lett., in press, July, 2003; www.aias.us

9. M. W. Evans, Lecture Notes in O(3) Electrodynamics (World Scientific, Singapore, in preparation); first two lectures available on www.aias.us

10. D. J. Clements and M. W. Evans, Higher Symmetry Electrodynamics in Special and General Relativity (Kluwer Academic, Dordrecht, in preparation); www.aias.us

11. R. M. Wald, General Relativity (University of Chicago Press, 1984).

12. G. Stephenson, Mathematical Methods for Science Students (Longmans \& Green, London, 1968). 
13. B. F. Schutz, A First Course in General Relativity (Wiley, New York, 1972).

14. A. Einstein, The Meaning of Relativity (Princeton University Press, 1921).

15. T. E. Bearden, in Ref. 7, Vol. 119(2), and www.cheniere.org

16. DVD available from A. Craddock; see www.cheniere.org of the Sweet device.

17. J. B. Marion and S. T. Thornton, Classical Dynamics of Particles and Systems (HBJ, New York, 1988).

18. D. Corson and P. Lorrain, Introduction to Electromagnetic Fields and Waves (Freeman, San Francisco, 1962). 